\title{
Etnografía en colaboración y representaciones sociales sobre infancia: Entre lo dicho y lo hecho
}

\section{Ethnography in collaboration and social representations about childhood: from what is said to what is done}

\section{DOI: https://doi.org/10.32870/dse.v0i20.594}

\author{
Anny Bertoli*1
}

\begin{abstract}
Resumen
El artículo propone la reflexión sobre una experiencia de etnografía colaborativa con niños, niñas y jóvenes del movimiento Gestores de Paz en la ciudad de Bogotá, en el cual la investigadora se percató de sus propias representaciones sociales sobre infancia y del conflicto que tienen estas con la postura ética y teórica planteada por la etnografía colaborativa. Por esto, el texto tiene la finalidad de mostrar cómo la etnografía en colaboración le permite al investigador alumbrar esos sentidos comunes sobre infancia, que siguen vigentes y que pueden determinar su trabajo investigativo con los niños, niñas y jóvenes.
\end{abstract}

Palabras clave: Etnografía colaborativa - representaciones sociales sobre infancia - reflexividad - movimientos sociales de niños - estudios de infancia.

\begin{abstract}
The article proposes a reflection on an experience of collaborative ethnography with children and youths of the "Children as Peacemakers" social movement in Bogotá, Colombia. During her fieldwork, the researcher became aware of her own social representations about childhood and how these conflicted with some ethical and theoretical tenets of collaborative ethnography. For this reason, this paper aims to show how collaborative ethnography allows the researcher to shed light on popular wisdom about childhood that still exists and may influence research work with children and youths.
\end{abstract}

Key words: Collaborative Ethnography - social representation about childhood - reflexivity - children social movements - childhood studies

* Maestra en Educación por la Pontificia Universidad Javeriana de Bogotá. Estudiante del Doctorado en Educación. Universidad de Auckland, Nueva Zelanda.anny.bertoli@auckland.ac.nz

1 Agradezco a Camilo Bácares Jara por ser mi primer lector: "Respira y canta. Donde todo se termina abre las alas". Versos del poema: Así sea de Blanca Varela. 


\section{Sin embargo, no se preocupen, sabré respetar sus titubeos. Mientras más se expone uno, mayores probabilidades tendrá de sacar provecho de la discusión, y más amistosas serán, de ello estoy seguro, las críticas o las sugerencias (la mejor manera de "liquidar" los errores, y los terrores que a mendo los motivan, sería riéndonos de ellos todos juntos).}

Pierre Bourdieu

\section{Introducción}

En las últimas décadas, los estudios sobre infancia han sufrido una revolución copernicana ya que los significados atribuidos, tanto al niño como al concepto de infancia, fueron puestos bajo una lupa y han sido analizados desde una perspectiva constructivista y estructuralista, por cierto, contrarias a las posiciones ontológicas y epistémicas hegemónicas de los investigadores en relación con estos fenómenos (Lange y Mierendorff, 2009). Esto permitió que en el plano investigativo se hayan modificado, por un lado, la relación del adulto-investigador con el niño y, por otro, el rol del niño en las diferentes fases de la investigación. Desde una mirada epistemológi$\mathrm{ca}$, este viraje ha implicado repensar las metodologías implementadas, dando como resultado que muchos métodos y técnicas fueran adaptados a los más pequeños y se buscaran formas más amigables que favorecieran su participación (Hogan, 2005). Es así que la etnografía ha sido uno de los tantos métodos en los que ha repercutivo este cambio, resultando afectada por la ruptura señalada. Por ejemplo, James y Prout reconocen a la etnografía como una

metodología particularmente útil para el estudio de la infancia. Permite a los niños una expresión y una participación más directa en la producción de datos sociológicos, mientras que no pasa lo mismo con la investigación experimental o por las encuestas (1997: 8).

De hecho, la etnografía se diferencia de otras formas de investigación por su intención de comprender la comunidad "junto con los nativos" y no "sobre los hombros de los nativos" (Lassiter, 2005). Y es que desde los trabajos de Malinowski y Boas la etnografía adquirió un carácter colaborativo, dado que los investigadores empezaron a adentrarse en la vida cotidiana de las comunidades, a percibirse como participantes en busca del "punto de vista" del otro (Lassiter, 2005). Sin embargo, Lassiter también reconoce que la etnografía "mueve la colaboración desde su trasfondo y lo posiciona en el centro del escenario" (2005: 16), impulsando al investigador a repensar cada fase de la investigación en clave colaborativa, así como también la distribución de poder en la relación con los nativos. Además de esto, Joanne Rappaport (2007) plantea que la colaboración en la etnografía es

Diálo@os sobre Educación año 11 | número 20 | enero-junio 2020 | ISSN 2007-2171 
una experiencia transformadora. No solo afecta a las organizaciones con las que trabajan académicos externos [...] sino que también lleva a esos académicos a repensar su propio oficio, a ir más allá de la academia en busca de teoría y método, [...] y repensar la forma en que se reflexiona sobre sí mismos y los demás (2007: 39).

La etnografía en colaboración, entonces, por un lado se caracteriza por la voluntad del investigador de compartir la perspectiva y la autoridad con los otros y en estar abierto a resultados que no habían sido contemplados (Hinson, citado por Lassiter, 2005); por otro lado, favorece una práctica reflexiva del investigador que es invitado a repensar su relaciones y acciones en el campo.

Por ello, la etnografía en colaboración explora las posibilidades de coproducir investigaciones con los niños, niñas y jóvenes (NNJ), quienes son reconocidos como co-investigadores y co-productores de conocimiento. Con este enfoque se reduce la distancia entre adulto y NNJ y se impulsan relaciones de poder compartido (Guerrero, Clemente, Milstein y Dantas-Whitney, 2017). La investigación que se presenta en esta ocasión está inserta en este marco teórico. Sin embargo, durante el trabajo de campo se evidenció que en algunas ocasiones las investigadoras realizaban acciones y comprensiones que podrían ser interpretadas como orientadas por un pensamiento adultocéntrico (Duarte Quapper, 2012). Reflexionando sobre las razones de lo que ocurría en dichas situaciones, se reconocieron ideas inconscientes y automáticas sobre infancia, que de alguna forma respondían a representaciones sociales históricas y de sentido común acerca de los NNJ.

De este modo, el presente artículo tiene como finalidad recoger las dudas, preguntas y reflexiones que surgieron durante el trabajo de campo de la investigación "Niños, niñas y jóvenes en movimiento. La configuración de la subjetividad política en la experiencia educativa y de participación política del movimiento Gestores de Paz", de Alexandra Barbosa Carrero y Anny Bertoli. En particular, en este texto se exponen algunas de las representaciones sociales sobre la infancia propuestas en la bibliografía, se retoman algunos acontecimientos de matriz adultocéntrica que tuvieron lugar, para luego analizarlos y mirar cómo la etnografía colaborativa favoreció su reconocimiento y reflexión.

\section{El contexto}

La investigación se desarrolló con un grupo de NNJ colombianos que forman parte del Movimiento Gestores de Paz (MGP), un movimiento social conformado por NNJ de diferentes edades, desde los más pequeños de 4 años a los jóvenes de 22 años, y tiene su origen en el Movimiento de Niños y Niñas por la Paz. Este último surgió a finales de la década de los noventa en Colombia, gracias a la colaboración que se tejió entre niños y niñas y varias organizaciones no gubernamentales, con el fin de impulsar un llamado de atención a lo que estaba pasando en el país al 
acentuarse el conflicto interno armado entre las FARC-EP, el ELN, los paramilitares y el Estado. La estrategia del movimiento fue promover una jornada de votación para que niños y niñas entre 7 y 17 años pudieran escoger los derechos que consideraban más importantes. El resultado fue sorprendente: 2 millones 700 mil niños y niñas salieron a la calle a votar y el derecho a la paz fue el más votado. A pesar de ese logro histórico, el Movimiento por la Paz no tuvo los recursos económicos, materiales y humanos necesarios para garantizar su continuidad. Frente a esto, a finales de la década se crearon nuevas experiencias inspiradas en la anterior, aunque más pequeñas y vinculadas a una organización social específica; este fue el caso del Movimiento de Niños, Niñas y Jóvenes Gestores de Paz, impulsado por la organización no gubernamental Visión Mundial en varias regiones de Colombia.

De este modo, la investigación se realizó con un grupo de NNJ de la ciudad de Bogotá, en el Potosí, un barrio empinado en los cerros del sur de la capital colombiana con calles inacabadas y servicios públicos precarios. Es un territorio que presenta problemáticas sociales vinculadas con el microtráfico, la violencia, la contaminación ambiental y la explotación minera, pero que también goza de una vigorosa vida cultural y social impulsada por diferentes organizaciones arraigadas en la comunidad.

La presencia del MGP en el barrio se remonta a 2006, cuando se forma el primer grupo de niños acompañado por un trabajador social de Visión Mundial. Actualmente, el MGP de Potosí está organizado en dos grupos: el de reciente ingreso y el de los mentores, NNJ encargados de llevar a cabo los talleres de formación con los grupos nuevos, llamados semilleros. Los talleres de formación se enfocan en varios temas relacionados con los derechos, el medio ambiente, la construcción de paz, etcétera.

Cabe agregar que la investigación se llevó a cabo desde febrero hasta finales de agosto de 2016, con la colaboración de los NNJ del grupo de mentores, con quienes se estableció una relación más cercana y duradera que permitió decidir los métodos de investigación, así como escribir una ponencia de forma colaborativa, la cual fue presentada en la II Bienal Iberoamericana de Infancias y Juventudes, organizada por la Universidad de Manizales y por el Cinde en noviembre de 2016. De aquí surge este trabajo.

En cuanto la investigación en colaboración, es un enfoque que favorece la reflexión acerca de las categorías presentes en los investigadores, que en este caso permitió la toma de conciencia de las representaciones sociales acerca de la infancia instauradas en quien escribe y que al fin y al cabo, de forma oculta inciden en la interpretación de la realidad, incluyendo la forma en que se realiza una investigación y qué se consigna en ella.

\section{Las representaciones sociales sobre infancia}

En los últimos treinta años se ha discutido bastante sobre la manera en que se percibe y considera al niño y a la categoría infancia, haciendo especial énfasis en cómo esto determina la forma

Diálo@os año 11 | número 20 | enero-junio 2020 | ISSN 2007-2171 
de relación generacional y todas las prácticas sociales, jurídicas y culturales destinadas a los más pequeños. $Y$ es que los significados atribuidos a lo que llamamos infancia favorecen o impiden formas de actuar y de vivir de los niños y las niñas, permiten o prohíben su participación en espacios públicos y le asignan un lugar real y simbólico en la sociedad (Bácares, 2012; Casas, 1998; Cussiánovich, 2010). Comúnmente, la infancia es asociada de manera inversa a la adultez, es decir, se piensa como una relación entre opuestos. Bustelo (2005) evidencia esta dicotomía planteando lo que él llama "la asimetría adultocéntrica", que se basa en algunos términos de comparación-disminución del niño como los siguientes: maduro/inmaduro, racional/emocional (irracional), autónomo/dependiente, mayor/menor, autoridad/obediencia, competente/ incompetente, completo/incompleto, público/privado, cultura/naturaleza, independiente/dependiente, trabaja/juega, actor/objeto, visible/invisible, fuerte/vulnerable, formado/maleable.

Podemos decir que, como lo han mostrado los estudios desarrollados en el campo sociológico, histórico y antropológico en las últimas décadas, estos significados resultan una producción social, histórica y política que se da con el trascurso y el cambio de los tiempos. Así, dichas representaciones sociales (Cussiánovich, 2010) tienen un largo recorrido en nuestras prácticas e interpretaciones, incluso, un papel fundamental en la determinación ontológica y epistemológica de la investigación referida a la infancia (Hogan, 2005; Matthews, 2007).

Por esta razón, en este apartado se enunciarán algunas de las representaciones sociales vinculadas con la categoría infancia, y en un segundo momento se analizará su conflicto y emergencia con la investigación y cómo la etnografía en colaboración, en cuanto enfoque reflexivo, contribuye a revelarlas.

Para empezar, es importante dejar asentado qué es una representación social. Moscovici, en su libro El psicoanálisis, su imagen y su público, la define como:

una modalidad particular del conocimiento, cuya función es la elaboración de los comportamientos y la comunicación entre los individuos [...] tiene una función constitutiva de la realidad, realidad que experimentamos y en la que nos movemos la mayoría de nosotros [...] La representación es un corpus organizado de conocimientos y una de las actividades psíquicas gracias a las cuales los hombres hacen inteligible la realidad física y social, se integran en un grupo o en una relación cotidiana de intercambios, liberan los poderes de su imaginación (1979: 17-18).

En otras palabras, las representaciones sociales son el conocimiento construido y naturalizado acerca de la realidad, que al mismo tiempo contribuye a la construcción de esa misma realidad. En el caso de las representaciones sociales sobre infancia, Ferrán Casas sostiene que tampoco la investigación y los investigadores sociales han sido inmunes a su influencia, por ejemplo, mostrando que en los estudios sobre socialización solamente se ha tomado en cuenta el rol de los padres y no la agencia de los niños y niñas en ese proceso; en los estudios judiciales 
se reproduce la imagen del niño como un ser incompetente, como un testigo no fiable; y en los estudios acerca de la calidad de vida, la opinión de niños y niñas no ha sido considerada, de hecho, la información acerca del tema ha sido propiciada por adultos, sean ellos "especialistas de infancia" o los padres (2006: 32-33).

Precisamente, en su texto Ensayos sobre Infancia II. Sujetos de Derechos y Protagonistas, Alejandro Cussiánovich (2010) describe algunas de las representaciones sociales dominantes a las que él llama culturas de infancia, a saber, "las distintas formas de pensar y de actuar que una sociedad reserva a sus nuevas generaciones [...] son asimismo una forma de sentir, de disponer nuestra afectividad, de adecuar nuestro cuerpo" (2010: 43).

Al respecto, una primera forma de pensar la infancia es la que el mismo Cussiánovich (2010) define como la del niño prescindible, esto es, la posibilidad del adulto de tomar decisiones sin consultarlo, tanto en el nivel político, educativo, social, cultural y económico. Generalmente, tampoco son consultados en el momento que se promulgan nuevas leyes o se deciden políticas sociales o económicas destinadas a ellos. De igual modo, en el ámbito escolar esa población carece de la posibilidad de decidir los contenidos y formas de aprendizaje o las reglas de convivencia, sobre todo en las edades más tempranas. Otro campo en el cual el niño ha sido considerado prescindible es el de la investigación. De hecho, solamente hasta 1960 se empieza a pensar que los NNJ pueden ser informantes en una investigación antropológica dado que —al igual que los adultos - pertenecen y conocen la sociedad en la que viven (Goodman, 1960). Posiblemente, una de las mayores manifestaciones de este ostracismo lo brindan las investigaciones que desechan el contexto de vida de los sujetos o que - en nuestro caso- no consideran el niño específico que pertenece a una infancia situada.

Por otra parte, en la representación social de la infancia patriarcal ocurre algo similar. En ella se considera al niño como un objeto o un subordinado; son los adultos "los únicos que deciden qué es bueno y qué deben hacer los niños [...] no se concede a los niños un ámbito propio" (Liebel, 2000: 41). Sin ambages, el niño no es valorado como un sujeto independiente y autónomo, no es merecedor de respeto físico o moral y los padres y los educadores tienen la facultad de utilizar el castigo físico para, supuestamente, corregir el comportamiento de los hijos o de los educandos (Cussiánovich, 2010). En el terreno de la investigación, la representación patriarcal de la infancia se evidencia en los estudios que consideran al niño casi como una rata de laboratorio (James y Prout, 1997; Green y Hogan, 2005; Kellett, 2011). En las décadas de los veinte y treinta, en la Universidad de Yale, Arnold Gessell inició los estudios de los niños en laboratorio (laboratory-based child study), poniendo particular atención en su desarrollo físico, social e intelectual (Woodhead y Faulkner, 2000). En este contexto, vale la pena mencionar que uno de los experimentos más famosos, conducido por Watson y Rayner, tenía el objetivo de mostrar — utilizando animales - que el miedo no es innato: se asustaba a un bebé con un peluche al mismo tiempo que se hacían ruidos. Lo mismo podemos hablar de la observación 
controlada que realizó Skinner con un bebé y su invento del baby box o la cuna área, donde dispuso del niño a su antojo (Woodhead y Faulkner, 2000; Lefrançois, 2000). Como se ve puede observar en estos experimentos, la voluntad y el bienestar del niño no fue considerada pues, una vez obtenido el permiso de los padres, el investigador podía actuar sin preocuparse de las consecuencias. En otras palabras, para relacionarse e investigar al niño, ese adulto necesitaba solo de un acuerdo con otro adulto para poner en marcha sus exploraciones.

Siguiendo con las representaciones propuestas por Cussiánovich, nos encontramos con la que él define como la del niño privatizado. Según esta postura, el niño es considerado como un sujeto que puede habitar solamente en el espacio privado de la familia o en un espacio tutelado por un adulto, impidiéndosele participar libremente en el espacio público y político (Bácares, 2012; Cussiánovich, 2010). Este mismo autor plantea que a pesar de la campaña actual para escuchar la voz de los niños, a ellos "se les ha quitado el peso y el sentido político de su participación" (2010: 51) puesto que el ejercicio se queda en escucharlos sin que sus planteamientos alcancen una incidencia o un eco político. En cierto sentido, lo mismo ocurre en lo que atañe a la investigación, porque la ruptura del niño actoral y con agencia que promocionó la sociología inglesa en muchos casos, solo sirvió para el avance metodológico o para la creación de nuevas técnicas de investigación amigables con los niños, en demérito del asunto central de ese proyecto: qué se hace con la información recolectada y si efectivamente esta influye en las decisiones de los investigadores o en las concernientes a otros aspectos. Igualmente, en muchas investigaciones la participación simbólica de los niños, como una forma de cumplimento de las indicaciones de la Convención sobre los Derechos de los Niños (CDN) o de las exigencias y parámetros éticos de estudio (Guerrero, et al., 2017), conduciría a un resultado parecido. En efecto, las grandes encuestas con recursos públicos estatales que indagan sobre el artículo 12 de la CDN o las investigaciones del Fondo de Naciones Unidas para la Infancia (Unicef, por sus siglas en inglés) ejemplifican a la perfección lo recién esbozado (Unicef, 2003). En México, por ejemplo, el Instituto Federal Electoral regularmente consulta a los NNJ mediante grandes encuestas para conocer sus preocupaciones y las maneras de mejorar sus derechos, pero ello no ha derivado en incidencia en políticas públicas ni en la aprobación de presupuestos, o sea, en tomar en cuenta sus opiniones (Van Dijk Kocherthaler, 2007).

En otro orden de ideas, en la representación clásica del niño inocente también hay mucho de aplazamientos y de prohibiciones para que los niños puedan expresarse a su gusto. Esta postura es muy antigua y se puede ubicar desde el cristianismo y la difusión de los Evangelios:

pura de corazón, simple y necesitada de amor: el concepto de infancia como "inocencia superior" y como "valor" representa una de las "latencias" de la cultura occidental, "uno de esos valores que son presentes a pesar de que no se ve" y que es destinado a aparecer nuevamente con nuevas energías (Di Bari, 2012: 27). 
Según Di Bari, esta tendencia se interrumpe con el Edicto de Constantino, en el año 313 d.C., cuando el niño empieza a ser considerado nuevamente como un sujeto marginal y reo del pecado original (citado por Di Bari, 2012). Tendrán que pasar varios siglos para que la idea de la infancia como inocencia vuelva a tomar vigor y se empiece a consolidar en el imaginario común. Justamente, autores como Boas, Cambi y Trisciuzzi (citados por Di Bari, 2012) ubican de nuevo a la infancia como manifestación de la inocencia durante el auge del empirismo; esta corriente consideraba a la experiencia y los sentidos del niño como naturalmente puros y todavía no contaminados por la cultura del hombre. No obstante, como bien se sabe, el mayor sostenedor y promotor del niño angelical fue sin duda Rousseau al atribuirle cualidades como la inocencia, la ingenuidad y la serenidad:

Amad la infancia, favoreced sus juegos, sus deleites y su ingenuo instinto. ¿Quién de vosotros no ha sentido deseos alguna vez de retornar a la edad en que la risa no falta de los labios y en la cual el alma siempre está serena? ¿Por qué queréis evitar que disfruten los inocentes niños de esos rápidos momentos que tan pronto se marchan, y de un bien tan precioso del que no pueden excederse? ¿Por qué queréis colmar de amarguras y dolores esos primeros años tan cortos, que pasarán para ellos y ya no pueden volver para vosotros? (Rousseau, 1973: 38).

Este mito de la infancia tomó tal fuerza con el romanticismo que en un sinfín de disciplinas y producciones — derecho, literatura, arte, filosofía, sociología, psicología, antropología y pedagogía - todavía podemos ubicarlo, y cómo no, analizarlo de cara a la investigación y al hecho de que los niños se expresen sobre su realidad. En efecto, dos ejemplos son suficientes para imaginar el calado limitante de esta proposición. Uno tiene que ver con los argumentos de los opositores de los derechos de los niños, en especial, el asociado a que es recomendable que los niños no sean portadores de derechos y de libertades puesto que estos son propios de los rigores de la vida adulta y perturbarían la candidez y ausencia de preocupaciones de los niños. De este modo, bajo este discurso los niños están imposibilitados de ejercer la palabra a menos que sea para aquello que fue delimitado estrictamente por los adultos como infantil y pueril:

La infancia es sinónimo de inocencia. Es la época cuando, prescindiendo de los rigores de la vida adulta, disfrutamos la libertad, experimentamos el juego y el gozo. El argumento reza: así como evitamos las responsabilidades y las adversidades de la vida adulta en la infancia, no debe haber necesidad de pensar en términos de derechos, un concepto que debemos asumir que está reservado para los adultos (Freeman, 2006).

Y el segundo, en un terreno más explícito y contradictorio, resulta una curiosidad total que uno de los principales momentos de promoción de los niños como informantes en las investi- 
gaciones derivara de la idea - o de sus ramificaciones - del niño como un recodo de inocencia. $Y$ es que, sin que esto opaque lo pionera que fue Mary Ellen Goodman al reconsiderar al niño en la investigación social como conocedor y como un miembro exclusivo de su cultura en dos de sus grandes textos (Goodman, 1957, 1960), Ilama la atención que en su defensa de los niños como informantes saliera a flote la mencionada representación de la inocencia. Lo que es interesante ver, ya que un paso tan crucial para los nacientes estudios sobre infancia fue basado también en la valoración de la mirada inocente de los niños:

Los niños pueden servir como informantes de tipo antropológico, siendo calificados como los adultos por la pertenencia a una sociedad y por el conocimiento de una parte limitada de la cultura de esa sociedad. Es razonable suponer que los niños no sólo pueden, sino que deben ser solicitados para actuar como informantes, ya que su (naiveté) ingenuidad/inocencia ofrece ventajas. Ellos nos pueden decir de primera mano y sin retrospección cómo parece su sociedad y su cultura desde sus ojos, o lo que la infancia es con respecto a sus percepciones de la sociedad y la cultura (Goodman, 1957: 979).

Desde otra perspectiva, una representación adicional de la infancia que goza de alto crédito es la del niño en desarrollo; una herencia que llega desde finales del siglo XVII cuando Locke escribió Algunos pensamientos sobre la educación (Di Bari, 2012). En este estudio propuso a la infancia como una fase de la vida que amerita una atención particular, pero también, que a lo largo de ella el niño se caracteriza por ser un sujeto pasivo y dependiente; imagen que se reforzaría a posteriori con la idea roussoniana de la necesidad de llegar a los 12 años para adquirir la capacidad de razonamiento (Hogan 2005). De tal manera, estos dos pensadores esbozaron las primeras percepciones del niño en desarrollo, idea que más tarde entrará definitivamente en el imaginario común con la popularización de la lectura de la obra de Piaget.

Siguiendo a Diane Hogan, en los estudios de esta corriente psicológica surge una representación del niño como un sujeto pasivo, dependiente y de poca confianza, que mantiene las mismas características de desarrollo progresivo en periodos históricos y lugares geográficos diferentes: "los niños son pensados, y por lo tanto investigados, como si ellos tuvieran una existencia que puede ser separada del contexto en que viven" (Hogan, 2005: 26). Así pues, la investigación influenciada por esta visión del niño tiende a descalificar las diferencias individuales, socioculturales e histórico-geográficas, y a privilegiar metodologías cuantitativas recolectando los datos mediante pruebas experimentales o de encuestas (Burman, 1994). Por ello, en su defecto, las metodologías cualitativas casi no son tomadas en consideración porque los niños son leídos como"informantes no fiables [...] ellos no pueden proporcionar información creíble y congruente/consistente acerca de los eventos o de las experiencias" (Hogan, 2005: 27); a pesar de que, en una contramirada, diversos estudios muestran que es mínima la distancia entre la pérdida de la memoria y la capacidad de recuerdo de los adultos y de los más pequeños (Spencer y Flin, 1990). 
Desde otro punto de vista, la asociación entre futuro y niñez, conducente a que los niños sean valorados por su utilidad social, tiene a la vez repercusiones en las formas de emprender investigaciones sobre la infancia. Pasa que la representación social de la potencialidad (Cussiánovich, 2010) sigue muy fecunda en los discursos de todos los días gracias a su largo recorrido. Por ejemplo, desde el siglo XV, la infancia ya había tomado un papel central en la sociedad dado que "los niños, escribe León Battista Alberti, tienen que ser direccionados hacia una juventud que sea ornamentada de virtudes y de buenos hábitos, por el bien de la familia" (Di Bari, 2012: 27). En la actualidad los niños son percibidos como una pieza dentro de una fórmula para el crecimiento económico y por eso se promulga con insistencia que hay que invertir dinero en su educación (Bustelo, 2005, Martínez Boom, 2011). Puntualmente, esta última posición ha tenido repercusiones en la hechura de investigaciones que tienen como principal objetivo conocer la experiencia de vida de los niños para mejorar su condición, y así apostar por un retorno económico en el futuro. Una prueba fehaciente de lo anterior la brinda el artículo Economic, neurobiological, and behavioral perspectives on building America's future workforce (Knudsen, Heckman, Cameron y Shonkoff, 2006) que se propuso demostrar cómo estudios económicos, neurocientíficos y de psicología del desarrollo plantean que las condiciones ambientales del sujeto durante la infancia influencian las capacidades y las posibilidades de logros en la edad adulta:

En pocas palabras, las habilidades generan habilidades, el éxito genera éxito y garantizar experiencias positivas en temprana edad es considerablemente menos costoso y más efectivo que la intervención correctiva en edad adulta [...] a medida que nos enfrentamos el tema de las necesidades de capital humano del futuro, los hallazgos de la neurociencia, de la investigación conductivista y de la economía coinciden en la siguiente conclusión: la prevención es más efectiva y menos costosa que el remedio, y temprano es mucho mejor que más tarde (Knudsen, Heckman, Cameron y Shonkoff, 2006: 41).

Ahora bien, tras esta rápida revisión de algunas de las ideas instaladas sobre los niños, se puede decir que muchas de ellas habitan en los diferentes actores que se relacionan con la infancia y que las mismas condicionan los puntos de vista sobre la forma de hacer investigación. En cierto modo, estas formas de percibir la infancia coexisten y se entrelazan creando combinaciones variadas en los discursos que producen y reproducen diariamente nuevas ideas acerca de cómo relacionarse con la niñez.

Al respecto, en las últimas cuatro décadas paulatinamente se ha dado un giro copernicano a estas representaciones con la creación de una nueva interpretación de la infancia y, por lo tanto, de cómo se concibe al niño. Este cambio es el resultado de avances y retrocesos en múltiples campos disciplinares que se dieron desde finales del siglo XIX y luego en el denominado "Siglo del Niño", en el que han jugado un papel crucial las coyunturas políticas, económicas y sociales 
de finales de la guerra fría, que crearon un clima favorable para la construcción de lo que hoy se conoce como la nueva representación social de la infancia.

En detalle, los años ochenta fueron el escenario de las reuniones anuales del Grupo de Trabajo sobre la Convención de los Derechos de los Niños hasta su proclamación en el 1989, de la creación del Norwegan Centre for Child Research (1982), pionero en los estudios de infancia; de la organización, por mano de Judith Ennew, del primer Ethnography Childhood Workshop en el King's College de Cambridge (1986), donde se conocieron los que hoy en día son los referentes en lengua inglesa de los Childhood Studies; del proyecto europeo Childhood as a Social Phenomenon (1987), coordinado por Qvortrup y Wintersberger, que tenía como objetivo el estudio de la infancia en 16 naciones y la creación de un grupo de trabajo internacional; y de la primera Cumbre Mundial en Favor de la Infancia (1990), en la cual 117 países elaboraron planes nacionales de acción por la infancia, que necesitaban de información y, por consiguiente, de investigaciones para poder desarrollarse (Bácares, 2012; Boyden y Ennew, 1997; Smith y Greene, 2012). A todo esto habría que agregar que en la década de 1990 ven la luz publicaciones que de algún modo recogen las reflexiones de la época y los cambios que se dan en la comprensión de la categoría, ya que por entonces aparecen textos escritos por William Corsaro, Jens Qvortup, Allison James, Adrian James, Pia Christensen, Chris Jenks y Alan Prout, entre otros (Smith y Greene, 2012).

De ahí que la categoría infancia empiece a ser considerada y socializarse como una construcción social y cultural y como un "elemento integrado y estructural en la organización de la vida social" (Qvortrup, 1987: 5), y que a partir de esta lectura las investigaciones empiecen a concentrarse en los procesos micro y el niño comience a ser visto como un actor social o un agente capaz y competente. Tales hallazgos, sumados a los aportes producidos en el campo jurídico, terminarían estableciendo una nueva representación del niño, que por un lado discute la idea del niño como incompleto, adulto en miniatura, futuro ciudadano o sujeto incapaz de entender la realidad que lo rodea, y por el otro, lo reconoce como un sujeto de derechos, sujeto político, agente y actor social, sujeto competente situado en el presente, y como un miembro activo capaz de reproducir e interpretar la cultura que habita.

De esto da fe la década de los noventa, cuando aparecen las primeras obras que vinculan el cambio de paradigma con la epistemología de la investigación. La más conocida es Children in Focus - A Manual for Participatory Research with Children", de Jo Boyden y Judith Ennew, donde se cuestiona la manera tradicional de hacer investigación con la infancia y se propone un manual para fomentar la participación de la niñez en la investigación. Del mismo modo, empezarían a surgir autores interesados en comprender las implicaciones del derecho a la participación de los niños en las investigaciones sociales (Woodhead y Faulkner, 2000; Alderson, 2000; Kellett, 2005).

En contraste con esto o yendo más allá, en los últimos años la nueva representación del niño, a la par ha permitido que se le mire como algo más que un informante, en concreto, como 
alguien capaz de producir conocimiento sobre su propia realidad al dotársele de herramientas y técnicas de recolección de información. Así, se ha explorado que el mismo niño sea el investigador de su propia realidad, con indagaciones hechas por ellos mismos (research by) como las que ha presentado el Children's Research Centre de la Open University del Reino Unido (Kellett, 2005).

Por el contrario, en América Latina, la emergente posición del niño ha dado paso al interés disciplinar por recoger sus voces, ante todo en los estudios históricos (Sosenski, 2016). Pero de manera novedosa y complementaria, en poco más de una década, también a la coproducción de conocimiento entre investigadores y niños a través de investigaciones con enfoque etnográfico en colaboración, en las cuales el niño es estimado como un coinvestigador que colabora en la construcción del saber. En particular, este enfoque está presente en los trabajos de los miembros de la Red Internacional de Etnografía con Niños, Niñas y Jóvenes (RIENN), fundada en 2008 por investigadores de diferentes países latinoamericanos como Diana Milstein (Argentina), Alba Lucy Guerrero (Colombia), Ángeles Clemente (México) y Michael Higgins (EE.UU y México).

Desde este último enfoque es que se desarrolló la investigación mencionada a inicio del texto. Sin embargo, este escrito tiene como finalidad mostrar cómo, a pesar del enfoque teórico y de este ángulo de la infancia actoral, en la tarea práctica de la investigación aparecen las demás representaciones, y cómo la etnografía en colaboración permite reflexionarlas y analizar sus conflictos en el devenir investigativo.

\section{Moverse entre representaciones}

En este apartado se reflexionará en torno a algunos episodios y decisiones en las que las representaciones relatadas en el acápite anterior prevalecieron, creando incomprensiones y actitudes adultocéntricas. Ante estas, vale subrayar que la atenta reflexión de los pensamientos y la escucha de los demás actores —en este caso de los NNJ_- ayudaron al reposicionamiento de la investigadora respecto de sus posturas iniciales.

Antes de comenzar, es pertinente aclarar que el campo se considera como una porción de la realidad que engloba tanto el espacio físico como el aspecto relacional, al igual que concentra significados y representaciones que los actores atribuyen al contexto, así como a los fenómenos sociales. Asimismo, es clave tomar en cuenta que el investigador trae consigo un universo de conceptos relacionados tanto con la teoría como con el sentido común (Gruber, 2001), especialmente en el caso de las investigaciones vinculadas con los NNJ, dado que la infancia, antes de ser una categoría teórica es una experiencia vivida por el investigador que a lo largo de su vida ha interiorizado y dotado de significados alrededor de este grupo social. De aquí que la reflexividad, inscrita en la labor etnográfica, se vuelva un instrumento indispensable para develar estas "categorías de pensamientos no pensados que delimitan lo pensable y predeterminan el pensamiento, y que guían la realización práctica del trabajo de investigación" (Bourdieu y Wacquant, 1995: 33).

Diólo@os

sobre Educación

TEMAS ACTUALES EN INVESTIGACION EDUCA año 11 | número 20 | enero-junio 2020 | ISSN 2007-2171 
De esta suerte, se considera cardinal dejar claro que la postura teórica de las investigadoras se inscribió en la corriente disciplinar que considera el niño como un actor social competente, sujeto de derechos, capaz de interpretar y producir conocimiento, pensado según los planteamientos de la nueva sociología de la infancia (James y Prout, 1997). De igual forma, desde el principio ambas investigadoras quisieron mantener una postura acorde con la voluntad y los tiempos de los chicos y con su derecho a ser investigados adecuadamente (Beazley, Bessell, Ennew y Waterson, 2009), es decir, se compartió con ellos los objetivos y la temática de la investigación, se pidió su opinión y su participación. De hecho, se decidió con ellos y ellas los tiempos y los espacios para realizar la investigación, así como los métodos para la recolección de los datos. La recolección de la información se realizó, por un lado, a través de la observación participante y la escritura del diario de campo, en el cual se reportaba tanto la descripción de los encuentros y los diálogos de los NNJ entre ellos y con las investigadoras, así como las "impresiones" de la misma investigadora al final del día. Por otro lado, se escogieron con los niños y niñas unas formas para poder conocer mejor sus vidas y la historia del movimiento. En este caso, los NNJ organizaron tres talleres: el primero fue un paseo por el barrio para mostrarnos y explicarnos la historia y las dinámicas sociales del lugar; en el segundo, nos mostraron sus fotografías personales y de su familia para compartirnos su historia de vida; y en el tercero, nos relataron la historia del MGP a través de una línea del tiempo desarrollada con el archivo fotográfico del movimiento. Cada taller fue grabado y transcrito. El material recogido durante el trabajo de campo (diarios, grabaciones y actas de las reuniones) fue analizado mediante la teoría fundamentada (Grounded Theory) de Strauss y Glaser, en particular porque este método "pone un gran interés en el desarrollo de una teoría sustentada en los datos procedentes del trabajo de campo" (Hernández Carrera, 2014: 192). De este modo, identificamos los códigos, y luego las categorías y subcategorías a partir de esos códigos, que permitieron definir discursos, imaginarios, significados y prácticas inherentes en los NNJ del Movimiento de Gestores de Paz de Potosí (Barbosa Carrero y Bertoli, 2016). El mismo método se utilizó para analizar el diario de campo en aras de encontrar las representaciones sociales sobre infancia instauradas en la investigadora.

De manera similar, la postura ontológica frente al niño era, sin duda, de investigadoras que reconocen a los más pequeños como agentes, sujetos con una perspectiva propia e inédita de la realidad, capaces de investigar y producir conocimiento de forma conjuntas; en otras palabras, los NNJ involucrados en la investigación fueron considerados como co-investigadores y co-productores. Al mismo tiempo, era claro que la infancia involucrada en la investigación participaba en procesos de formación política, a través de los cuales los niños conocen sus derechos, comprenden su actoría social y se perciben como sujetos públicos y transformadores de la sociedad (Cussiánovich, 2010). 
Ahora bien, dadas esas premisas fundamentales para advertir que todas las intenciones teóricas y ontológicas de las investigadoras dejaban de pensar en la ausencia de representaciones del niño o niña en las que prevaleciera el adultocentrismo o que los desconocieran como sujeto y actor social, se quiere mostrar cómo en ciertos casos las representaciones sociales exploradas a lo largo del texto siguieron vivas en la investigadora y cómo estas influenciaron las acciones e interpretaciones derivadas de la relación que se construyó con los chicos y en la comprensión de la realidad estudiada. Para hacer esto, se va reportando unos incidentes de campo particulares (Gruber, 2001) en los que brotaron esas infancias "clásicas", mostrando cómo en algunos casos las misma resultaron una limitación en la comprensión de la subjetividad de los NNJ.

El trabajo de campo con el MGP empezó a principios de febrero, cuando se realizaron los primeros acercamientos y los encuentros para conocernos y aclarar las razones de la presencia de las investigadoras y las dudas de los NNJ referidas al estudio; se recalcó mucho la intención de trabajar colaborativamente, de la mano con ellos, tanto para realizar la investigación como para poder apoyar sus actividades. Esos primeros meses fueron dedicados también a la comprensión de la organización del movimiento y de sus dinámicas participativas; es aquí donde surgen las primeras formas de adultocentrismo, de interpretación de la realidad de los niños sin los niños, ya que en el diario de campo, junto con las observaciones cotidianas, se relatan las primeras dudas acerca de la "real" participación de los niños más pequeños, de las formas de tomar decisión y de la organización de las actividades:

En la mañana tuvo formación con la mesa ambiental. El taller se desarrolló con dinámicas poco participativas. Los niños del MGP se reunieron en el patio del jardín y algunos integrantes de la mesa ambiental dieron el taller sobre cuidado del medio ambiente [...] los niños no participaron, simplemente contestaban preguntas cerradas (diario de campo, 27 febrero 2016).

Retomando estos apuntes y leyéndolos en busca de las representaciones plasmadas, es fácil entender que se estaba mirando a estos niños a través de la lente de la prescindibilidad, es decir, se miraba un contexto específico, a niños con un fuerte vínculo con su territorio, con una historia en el movimiento que todavía se desconocía, pero en comparación con otros niños de otra realidad sociopolítica conocidos en años anteriores en el Movimiento de Niños, Niñas y Adolescentes Trabajadores en Perú. Además, este parangón había sido construido también con las lecturas y las teorías sobre movimientos sociales en general, y de niños en particular. Inicialmente, la interpretación de lo observado prescindía de la experiencia de los NNJ presentes, de su opinión, de su historia como movimiento, de sus formas de entender el contexto y de las necesidades de este. El pasar del tiempo, y en el diálogo constante con los más pequeños, permitió entender que la organización del movimiento y la participación de los NNJ respondía 
a exigencias y características del entorno social y cultural, de la especificidad de la historia de Gestores de Paz.

Otro rasgo del niño como sujeto prescindible, latente en la última fase de la investigación, surgió cuando la información recolectada con los NNJ tenía que ser analizada e interpretada para llegar a escribir los resultados de la investigación. En esta etapa del estudio, los niños ni participaron ni fueron invitados a participar por dos razones principales: en un comienzo, por los tiempos académicos que difícilmente coinciden con los de la colaboración y, en segundo lugar, por la presencia de la idea del niño en desarrollo.

En detalle, la investigación se realizó en un tiempo que respondía al calendario académico de la Maestría en Educación de la Pontificia Universidad Javeriana; así, la duración del trabajo de campo, del análisis de los datos o de la escritura, no fueron libremente escogidos por las investigadoras y fueron compartidos con obligaciones laborales o de estudio. Por tal razón, la fecha de entrega del texto final no concordó con los tiempos de la colaboración con NNJ, que de por sí se caracterizan por ser dilatados, a veces confusos, inciertos y dependientes de los eventos propios del movimiento. Entonces, el análisis de datos y la escritura del texto respondió más a las coyunturas temporales referidas a la entrega de la tesis.

Paralelamente, otro factor influyó en tal decisión y en la idea de que el análisis de datos fuera una tarea difícil para niños que estaban estudiando la primaria o la secundaria, considerando que se necesitaba una serie de conocimientos teóricos y disciplinares específicos. De esta forma, se avaló la idea de que hay etapas de desarrollo y saberes que son reservados a los adultos, a pesar de referirse a los mismos niños. Más aún, se consideró innecesarios u opcionales los aportes de los NNJ en esta fase de la investigación en cuanto es "tarea del investigador adulto" analizar la información recolectada y vincularla a estudios académicos reconocidos por la comunidad científica. Lo anterior no quiere decir que la exclusión de los NNJ de esta fase haya sido una decisión tomada evaluando racionalmente estas variables, sino que, repensando y reflexionando el desarrollo de la investigación, la investigadora se dio cuenta que entre los múltiples factores que impulsaron tal modus operandi se encuentran las representaciones del niño en desarrollo y del niño prescindible que anidan en el mundo adulto.

Algo muy similar sucedió durante la decisión de trabajar en colaboración para la escritura de la ponencia para la II Bienal Iberoamericana de Infancias y Juventudes, mencionada anteriormente. La propuesta surgió desde la voluntad y el entusiasmo de escribir con los NNJ y no sobre su experiencia, en un intercambio de conocimientos y destrezas, colaborando en una actividad generalmente reservada a un grupo específico de adultos. La ponencia incluía cuatro tópicos caracterizados por niveles diferentes de abstracción: desde el más concreto, relacionado con las actividades que el MGP realiza en la comunidad, hasta la explicación del significado atribuido a la categoría construcción de paz. En esta ocasión, los niños y niñas con edades inferiores a los 
12 años se dedicaron a la descripción de las actividades concretas que el grupo de los mentores lleva a cabo con los demás niños, mientras que los mayores fueron los que se encargaron de relatar las partes más abstractas de la ponencia. En pocas palabras, en la subdivisión en grupos se vio reflejada la idea piagetiana del desarrollo cognitivo, la cual presupone que "hay un cambio desde un pensamiento fragmentado, parcial, concreto y estrechamente vinculado a la experiencia típica de la infancia, a un pensamiento lógico, abstracto y muy flexible de la adolescencia" (Meadows, 2018: 92). A pesar de esto, durante uno de los encuentros para relatar la ponencia, Sol, de 7 años, afirmó que una de las actividades desarrolladas por el MGP era importante para que "no se discrimine a las niñas. Los niños aprendan que las niñas pueden hacer lo mismo que ellos" (diario de campo, 26 agosto 2016). En palabras diferentes, estaba comunicando que el objetivo final de una de las actividades tenía la intención de trasformar formas de pensamiento abstracto, no solo de realizar acciones concretas e inmediatas. Sus palabras, refiriéndose a un problema social de amplia envergadura, alumbraron y al mismo tiempo desarbolaron la inconsciente representación del niño en desarrollo de la investigadora, esto es, la idea de que a una forma de lenguaje poco complejo corresponda la falta de capacidad de abstracción, cuestión ya resuelta en 1979 por Margaret Donaldson en su famoso libro La mente de los niños, cuando expuso algunas críticas a la teoría del desarrollo cognitivo afirmando que de manera generalizada se confunde la habilidad lingüística del niño con su habilidad intelectual en general. Otra ocasión, fue el aporte de los niños y el retomar ese evento lo que permitió entender cómo los hábitos rutinarios de pensamiento de la investigadora estaban influenciando la manera de relacionarse y actuar con los NNJ.

Justamente, fue durante un recorrido por el barrio con los NNJ que la representación del niño como inocente, ingenuo, que actúa sin considerar las posibles malas intenciones del otro o la complejidad de una situación o de un lugar, se concretó al juzgar las palabras de Luz como "muy bonitas" y al pensar que "no se da cuenta de lo que la rodea". Resulta que durante el recorrido, Jazmín, de 10 años, utilizó las palabras "mi mundo Potosí" para describir el lugar donde vive, una forma que entra al oído como si fuera el comienzo de un cuento de hadas o por lo menos así fue percibido por la investigadora cuando la escuchó hablar en estos términos por primera vez, describiendo su barrio de una forma muy positiva:

Pues a mí me gusta Potosí, y Potosí para mí es un hogar, es mi hogar, es mi familia, es el lugar donde me acogen, donde me cuidan, donde me aman, donde por mí todos me conocen saben de mí, todos me protegen todos me aman como yo soy y en mi Potosí todo es posible (Barbosa Carrero y Bertoli, 2016).

Relato que chocaba con la percepción del territorio: servicios precarios, zonas de microtráfico, altos índices de muertes violentas, condiciones ambientales insalubres por la fuerte contaminación, etc. Sin embargo, todo quedó grabado, y en un segundo momento se puede volver a sus palabras 
analizándolas detalladamente. Leyendo la transcripción de la conversación con Jazmín, fue claro que esta categoría de "mi mundo Potosi" rebosaba de significados que iban más allá de una percepción emocional del barrio por parte de los NNJ, de una visión ingenua o inocente de un territorio difícil. Los NNJ veían con claridad la complejidad del lugar, reconocían los aspectos positivos y los negativos: "y pues por seguir hablando de mi mundo Potosí, sería como un mundo con juegos, aunque con peligro, pero con juegos, o sea, no mucho peligro, aunque a ti te cuenten que Ciudad Bolívar es mucho peligro, mucho, mucho peligro, no lo creas". Los NNJ reconocen que en el barrio hay peligro, "por allí es mejor no andar: pueden robarlo a uno, es donde se meten vicio,", pero también reconocen que hay vecinos solidarios y espacios como el MGP, donde ellos pueden jugar, disfrutar del tiempo juntos, crear vínculos y amistades. Son bien conscientes que la representación de los demás, de los que no viven en el barrio o en la localidad, se relacionan con la peligrosidad, y por eso Jazmín sugirió no creer a los que afirman que hay "mucho, mucho peligro", es decir, a quien desconoce ambas facetas. Como se puede ver, Jazmín estaba ofreciendo unas claves de lectura complejas acerca de su barrio, que por la presencia oculta de la idea del niño como un ser angelical o"bonito", en un primer momento no fue posible reconocerlas y profundizarlas de forma más pertinente.

Así las cosas, estos ejemplos sirven para mostrar cómo todavía conviven y son concurrentes, a pesar de las reflexiones teóricas, las categorías "clásicas" de la infancia en la relación con el niño, a veces en la interpretación de sus relatos o en las metodologías utilizadas en la investigación.

\section{Conclusiones}

En este artículo se presentaron inicialmente algunas de las representaciones sociales históricas que se han relacionado con la infancia y han sido desarrolladas tanto en la literatura producida en el hemisferio norte como por autores de Latinoamérica. En un segundo momento, se discutieron algunos eventos que denotan el conflicto entre reconocer a los niños como actores sociales y el adultocentrismo que tuvo lugar en la investigación "Niños, niñas y jóvenes en movimiento. La configuración de la subjetividad política en la experiencia educativa y de participación política del movimiento Gestores de Paz", de Alexandra Barbosa Carrero y Anny Bertoli.

La reflexión acerca de los diferentes momentos de la investigación y del trabajo de campo muestran - a pesar del marco teórico y la etnografía en colaboración- actitudes y comportamientos de la investigadora que se enmarcan en el adultocentrismo, es decir, en un modelo relacional guiado por representaciones sociales acerca de los NNJ, que se caracterizan por enraizarse en definiciones y percepciones de control, incapacidad, etc., que siguen presentes en el nivel social y cultural en nuestras prácticas cotidianas con los niños.

2 Uso de sustancias psicoactivas. 
De tal manera, se ha necesitado regresar a estos eventos e intentar comprender cuáles habían sido las representaciones sociales heredadas que influían en las interpretaciones y decisiones de la investigadora, dando crédito de igual modo a cómo la misma presencia y discusiones de los niños fueron las que permitieron reconocer estos hábitos mentales referidos a la infancia.

En síntesis, la etnografía en colaboración y la respectiva reflexividad permitieron reconocer categorías ocultas que intervienen, atraviesan y direccionan la investigación y la relación con los NNJ.

\section{Bibliografía}

Alderson, P. (2000). Children as Researchers: The Effects of Participation Rights on Research Methodology. En P. Christensen y A. James (eds.). Research with Children. Perspectives and Practices (pp. 241-257). Nueva York: Routledge Falmer Press.

Bácares, C. (2012). Una aproximación hermenéutica a la Convención sobre los Derechos del Niño. Lima: Ifejant.

Barbosa Carrero, A. y A. Bertoli (2016). Niños, niñas y jóvenes en movimiento. La configuración de la subjetividad política en la experiencia educativa y de participación política del Movimiento Gestores de Paz. (Tesis de Maestría). Pontificia Universidad Javeriana, Bogotá, Colombia.

Beazley, H., S. Bessell, J. Ennew y R. Waterson (2009). The Right to be Properly Researched: Research with Children in a Messy, Real World. Children's Geographies, 7(4), 365-378.

Boyden, J. y J. Ennew (1997). Children in Focus - A Manual for Participatory Research with Children.

Estocolmo: Save The Children

Bourdieu, P. y L. J. D. Wacquant (1995). Respuestas. Por una antropología reflexiva. México: Grijalbo. Burman, E. (1994). Deconstructing Developmental Psychology. Londres: Routledge.

Bustelo, E. (2005). Infancia en indefensión. Salud Colectiva, 1(3), 253-284.

Casas, F. (1998). Infancia: perspectivas psicosociales. Barcelona: Paidós.

Cussiánovich, A. (2010) Ensayo sobre infancia Il. Sujeto de derechos y protagonista. Lima: Ifejant.

Di Bari, C. (2012). L'infanzia tra realtà, mito e immaginario. En Cambi, F., C. Di Bari y D. Sarsini II mondo dell'infanzia. Dalla scoperta, al mito, alla relazione di cura. Autori e testi. Milán: Apogeo, 21-54.

Donaldson, M. (1979). La mente de los niños. Madrid: Morata.

Duarte Quapper, C. (2012). Sociedades adultocéntricas: Sobre sus orígenes y reproducción. Última Década, (36), 99-125.

Freeman, M. (2006). Tomando más en serio los derechos de los niños. Revista de Derechos del Niño, (3-4), 251-279.

Goodman, M. E. (1957). Values, Attitudes, and Social Concepts of Japanese and American Children. American Anthropologist, 59(6), 979-999.

Diálo os 
(1960) Children as Informants: The Child's-Eye View of Society and Culture. The American Catholic Sociological Review, 21(2), 136-145.

Greene, S., y D. Hogan (2005). Researching Children's Experience. Londres: SAGE Publications.

Guber, R. (2001). La etnografía. Método, campo y reflexividad. Bogotá: Grupo Editorial Norma.

Guerrero, A. L., A. Clemente, D. Milstein y M. Dantas-Whitney (2017). Bordes, límites y Fronteras. Encuentros etnográficos con niños, niñas y adolescentes. Bogotá: Pontificia Universidad Javeriana.

Hernández Carrera, R. M. (2014). La investigación cualitativa a través de entrevistas: Su análisis mediante la Teoría Fundamentada. Cuestiones Pedagógicas, 23, 187-210.

Hogan, D. (2005). Researching 'the Child' in Developmental Psychology. En Greene, S. y D. Hogan. Researching Children's Experience. Londres: SAGE Publications, 22-41.

Kellett, M. (2011). Researching with and for Children and Young People. Centre for Children and Young People Background Briefing, (5). http://epubs.scu.edu.au/cgi/viewcontent. cgi?article $=1044 \&$ context=ccyp pubs

Knudsen, E., J. Heckman, J. Cameron y J. Shonkoff (2006). Economic, Neurobiological and Behavioral Perspectives on Building America's Future Workforce. World Economics, 7(3), 17-41.

James, A. y Prout, A. (ed.). (1997). Constructing and Reconstructing Childhood. Contemporary Issues in the Sociological Study of Childhood. Londres: Routledge Falmer Press.

Lange, A. y J. Mieredorff (2009). Method and Methodology in Childhood Research. En J. Qvortrup. W. Corsaro y M. S. Honig, The Palgrave Handbook of Childhood Studies. Londres: Palgrave Macmillan, 78-96.

Lassiter, L. E. (2005). The Chicago Guide to Collaborative Ethnography. Chicago: The University of Chicago Press.

Liebel, M. (2000). La otra infancia. Niñez trabajadora y acción social. Lima: Ifejant.

Martínez Boom, A. (2011). Unicef ... dejad que los niños vengan a mí. Revista Educación y Pedagogía, 23(60), 45-64.

Matthews, S. H. (2007). A Window on the "New" Sociology of Childhood. Sociology Compass, 1(1), 322-334.

Meadows, S. (2018). Understanding Child Development: Psychological Perspectives and applications. Nueva York: Routledge.

Qvortrup, J. (1987). Introduction. International Journal of Sociology, 17(3), 3-35.

Rappaport, J. (2007). Anthropological Collaborations in Colombia. En Field, L. y R. Fox (eds.). Anthropology put to Work. Oxford/New York: Berg, 21-44.

Smith, C. y S. Greene (2014). Key Thinkers in Childhood Studies. Bristol: Policy Press.

Sosenski, S. (2016). Dar casa a las voces infantiles: Reflexiones desde la historia. Revista Latinoamericana de Ciencias Sociales, Niñez y Juventud. 14(1), 43-52.

Spencer, J. R. y R. Flin (1990). The Evidence of Children: The Law and the Psychology. Londres: Blackstone Press. 
Woodhead, M. y D. Faulkner (2000). Subjects, Objects or Participants? Dilemmas of Psychological Research with Children. En P. Christensen y A. James (eds.). Research with Children. Perspectives and Practices. Nueva York: Routledge Falmer Press, 9-36.

UNICEF (2003). Estado Mundial de Ia Infancia. Nueva York: autor.

Van Dijk Kocherthaler, S. C. (2007). Participación infantil. Una revisión desde la ciudadanía. Tramas (28), 48-66. 